\title{
Autonomic cardiac regulation during spontaneous nocturnal hypoglycemia in children with type 1 diabetes
}

\author{
Sara Bachmann ${ }^{1,2}$ ( ) | Anne Auderset ${ }^{3}$ | Marie-Anne Burckhardt ${ }^{1,2}$ ( ) | \\ Gabor Szinnai $^{1,2}$ | Melanie Hess ${ }^{1}$ | Urs Zumsteg ${ }^{1}$ | Kris Denhaerynck ${ }^{4}$ | \\ Birgit Donner ${ }^{3}$
}

\author{
${ }^{1}$ Pediatric Endocrinology and Diabetology, \\ University Children's Hospital Basel, Basel, \\ Switzerland \\ ${ }^{2}$ Department of Clinical Research, University \\ of Basel, Basel, Switzerland \\ ${ }^{3}$ Pediatric Cardiology, University Children's \\ Hospital Basel, Basel, Switzerland \\ ${ }^{4}$ Department of Public Health, Institute of \\ Nursing Science, University of Basel, Basel, \\ Switzerland

\section{Correspondence} \\ Sara Bachmann, Pediatric Endocrinology and \\ Diabetology, University Children's Hospital \\ Basel, Switzerland. \\ Email: sara.bachmann@ukbb.ch
}

\begin{abstract}
Background: Hypoglycemia is the most common complication in insulin treated diabetes. Though mostly mild, it can be fatal in rare cases: It is hypothesized that hypoglycemia related QTc prolongation contributes to cardiac arrhythmia.

Objective: To evaluate influence of nocturnal hypoglycemia on QTc and heart rate variability (HRV) in children with T1D.

Methods: Children and adolescents with T1D for at least 6 months participated in an observational study using continuous glucose monitoring (CGM) and Holter electrocardiogram for five consecutive nights. Mean QTc was calculated for episodes of nocturnal hypoglycemia ( $<3.7 \mathrm{mmol} / \mathrm{L}$ ) and compared to periods of the same duration preceding hypoglycemia. HRV (RMSSD, low and high frequency power LF and HF) was analyzed for different 15 min intervals: before hypoglycemia, onset of hypoglycemia, before/after nadir, end of hypoglycemia and after hypoglycemia.

Results: Mean QTc during hypoglycemia was significantly longer compared to euglycemia ( $412 \pm 15$ vs. $405 \pm 18 \mathrm{~ms}, p=0.005$ ). HRV changed significantly: RMSSD (from $88 \pm 57$ to $73 \pm 43 \mathrm{~ms}$ ) and HF (from $54 \pm 17$ to $47 \pm 17 \mathrm{nu}$ ) decreased from before hypoglycemia to after nadir, while heart rate (from $69 \pm 9$ to 72 $\pm 12 \mathrm{bpm}$ ) and LF (from $44 \pm 17$ to $52 \pm 21 \mathrm{nu}$ ) increased ( $p=0.04$ ).

Conclusion: A QTc lengthening effect of nocturnal hypoglycemia in children with T1D was documented. HRV changes occurred even before detection of nocturnal hypoglycemia by CGM, which may be useful for hypoglycemia prediction.
\end{abstract}

\section{KEYWORDS}

diabetes, ECG, heart rate variability, hypoglycemia, QTC

\section{1 | INTRODUCTION}

Hypoglycemia is an unavoidable and dreaded acute complication of insulin treated diabetes mellitus. Although most nocturnal hypoglycemic episodes are mild and asymptomatic, they can be fatal in rare cases: Sudden unexpected death in otherwise healthy young people with type 1 diabetes (T1D) has been reported to be up to 10 times higher compared to a population without diabetes. ${ }^{1,2}$ It is hypothesized that these overnight deaths (so called "dead in bed phenomenon") are induced by hypoglycemia. ${ }^{3,4}$ The precise mechanism by 
which hypoglycemia leads to death is still not fully understood. There is some evidence that insulin-induced hypokalemia in combination with sympatho-adrenergic stimulation leads to fatal cardiac ventricular arrhythmia in adults. ${ }^{5}$

Even episodes of mild or moderate hypoglycemia seem to have an impact on cardiac repolarization: In adults with ${ }^{6-9}$ and without diabetes $^{5,10}$ lengthening of QTc intervals during experimental and spontaneous hypoglycemia has been documented.

In a previous study, we observed frequent nocturnal hypoglycemia in children with T1D, often prolonged for hours. ${ }^{11}$ Hence, the question remains, whether alterations of QTc intervals occur during nocturnal hypoglycemia in children with diabetes.

Besides QTc intervals, cardiac regulation is represented by heart rate variability. Heart rate variability measures the beat-tobeat fluctuation of heart rate and represents the interplay of the sympathetic and parasympathetic nervous system. ${ }^{12}$ Because hypoglycemia stimulates the sympathetic nervous system, a reaction of heart rate variability during episodes of hypoglycemia can be assumed. In adults, such hypoglycemia-induced changes in heart rate variability have already been reported. ${ }^{7,9,13}$ It is hypothesized that these changes may be helpful in the prediction of hypoglycemia. ${ }^{13,14}$ However, in children with T1D, the effect of hypoglycemia on heart rate variability has not been studied so far.

Therefore, aims of this study were to investigate if nocturnal hypoglycemia results in QTc prolongation and changes in heart rate variability in children with T1D.

\section{2 | RESEARCH DESIGN AND METHODS}

\section{1 | Participants}

Children and adolescents aged below 18 years with T1D for at least 6 months and followed at our outpatient department were recruited. Participants with pre-existing cardiac disease or medication known to affect cardiac function were excluded. Participants aged 14 years and older and all parents of the participants were asked to provide written informed consent.

The study was approved by the local ethics committee (BASEC 2016-01772).

\section{2 | Study design}

We performed a prospective observational study in children with T1D in the home setting.

After assessment of baseline data (HbA1c, weight, height, age, diabetes duration, insulin treatment/dosage) participants underwent a 5-day monitoring period: Blinded continuous glucose monitoring (CGM) was performed during five consecutive days and nights and combined with Holter electrocardiogram (ECG) recording during every night. Participants continued with their routine insulin treatment and daily activities and were instructed to perform at least three capillary blood glucose measurements per day for calibration and to record injected insulin doses, consumed carbohydrates, bed- and wake-up time and hypoglycemic symptoms in a logbook. After 5 days, the devices were returned for data download.

\section{$2.3 \quad$ Baseline assessments}

$\mathrm{HbA1c}$, weight, height, insulin treatment and insulin dose (units/kg/day and percentage of basal insulin) were documented at the routine visit preceding the study. All participants were seen by a cardiologist and cardiac disease (such as congenital or acquired structural heart disease, heart insufficiency or clinically relevant arrhythmia) was excluded. At the start of the measurement period, a capillary blood sample was taken for the analysis of serum calcium, potassium and magnesium.

\section{4 | Continuous glucose monitoring}

Glucose was measured by a blinded CGM system (iPro Transmitter and Enlite sensor, Medtronic Minimed, Northridge, CA). The system has proven accuracy in children with a mean absolute relative difference (MARD) of $10.1 \%$, and in the range of $2.22-4.44 \mathrm{mmol} / \mathrm{L}$ a sensor-to blood glucose agreement of $85.5 \%$ has been reported. ${ }^{15}$ If participants were already using a CGM system (Dexcom or Medtronic), CGM data from these devices were used for analysis. The performance of the Dexcom G4 sensor is comparable to the Enlite sensor (MARD 11\%, accuracy in hypoglycemia 80\%). ${ }^{16,17}$

\section{5 | Electrocardiogram}

A three channel Holter ECG (Schiller, FD12plus) was mounted by the participants and parents every evening and worn at nighttime (from bed time to wake up time) for at least five consecutive nights. The participants/their families were educated on the use of the Holter ECG and written and illustrated instructions were handed out.

\subsection{CGM analysis}

Hypoglycemia was defined as interstitial glucose below $3.7 \mathrm{mmol} / \mathrm{L}$ for at least $15 \mathrm{~min}$. A hypoglycemic event was considered symptomatic, if hypoglycemic symptoms were documented in the logbook.

\subsection{ECG analysis}

All ECG recordings were screened by a cardiologist and artifacts were excluded. Excluded beats were not replaced by interpolated beats. ECGs were transferred to MATLAB (MathWorks, Natick, MA) and Labchart (Version 8, AD Instruments, Sidney, AU) for further analysis. 
QTc was calculated by the Bazett formula (QT divided by the square root of the RR interval). For every episode of hypoglycemia, mean QTc of the entire episode was calculated and compared to a preceding interval of the same duration. To evaluate the effect of sensor glucose on QTc intervals, all recorded sensor glucose values were correlated to the corresponding QTc intervals ( 5 min means). To consider a possible time delayed effect of glucose on QTc, glucose level with time shifted QTc intervals (from 15 to $225 \mathrm{~min}$ ) were compared.

\subsection{Heart rate variability}

After exclusion of artifacts, RR intervals were identified automatically by the LabChart software, and heart rate variability parameters according to standards ${ }^{18}$ were calculated.

Time domain analysis included standard deviation of the RR interval (SDRR) and square root of the mean standard differences of successive RR intervals (RMSSD). SDRR represents the overall variability and cannot be assigned to a specific part of the autonomous nervous system, whereas RMSSD indicates short-term variability and represents parasympathetic activity.

Frequency domain analysis was performed by fast Fourier transformation to analyze the parameters low frequency power (LF, 0.04$0.15 \mathrm{~Hz}$ ) and high frequency power (HF, 0.15-0.4 Hz). HF reflects parasympathetic activity, whereas LF is considered to be a marker of sympathetic modulation.

Heart rate variability (HRV) parameters were calculated in $15 \mathrm{~min}$ windows before the onset of hypoglycemia, at the start of hypoglycemia, before nadir, after nadir, at the end of hypoglycemia and for the first $15 \mathrm{~min}$ after a hypoglycemic episode.

\subsection{Statistical analysis}

Baseline data and hypoglycemic episodes were described using frequencies (\%), means $( \pm \mathrm{SD}$ ) or medians (range) as appropriate. Differences between average QTc intervals before and during hypoglycemic episodes were compared by random-intercepts regression analysis (using patient as a random variable). Possible moderation by variables such as sex, age, diabetes duration, $\mathrm{HbA1c}$, duration and nadir of hypoglycemia and serum level of calcium, potassium and magnesium was examined by testing interaction effects between the candidate moderator and the episode (hypoglycemic episode yes/no).

To estimate the effect of glucose values on concurrent and subsequent QT intervals, we employed the same analysis method (i.e., randomintercepts regression analysis with patient as a random variable).

Heart rate variability parameters over time were estimated by random-intercepts regression analysis, using episodes as random variable. For each parameter, two separate models were tested: a first from before hypoglycemia to after nadir; and a second from before nadir to after hypoglycemia. Analyses were performed using SAS 9.4 (SAS Institute, Cary, NC).

\section{3 | RESULTS}

\section{1 | Participant characteristics}

Twenty-five children (44\% female) aged $8.1-17.6$ years (mean age 13.5 years, SD 2.5 years) were included in the study. Diabetes duration ranged from 0.5 to 13.3 years. Fourteen participants (56\%) used continuous subcutaneous insulin infusion, $\mathrm{HbA} 1 \mathrm{c}$ ranged from 6.3 to $12.9 \%$ (mean $7.8 \%$, SD 1.4\%).

See Table 1 for an overview of baseline characteristics.

\section{2 | Recordings}

Complete simultaneous CGM and Holter ECG recordings for five nights were available in 13 patients ( $=65$ nights), 4 complete nights in another 8 patients ( $=32$ nights). In four children, more than one night of glucose or ECG was missing. A total of 106 nights with complete simultaneous recordings were available for analyses. Twelve participants used their own CGM (10 Medtronic, 2 Dexcom), 13 used the iPro system.

\section{3 | Hypoglycemia}

During 32 nights 42 episodes of nocturnal hypoglycemia occurred in 19 patients. Fourteen patients had repeated nocturnal hypoglycemia. Simultaneous ECG recordings were available for 34 hypoglycemic episodes in 17 patients, in 11 patients two or more episode were integrated in the analysis. In 16 (47\%) of those episodes nadir sensor glucose level was below $3.0 \mathrm{mmol} / \mathrm{L}$, in 18 episodes between 3.0 and $3.6 \mathrm{mmol} / \mathrm{L}$.

The duration of analyzed hypoglycemia events ranged from 15 to $365 \mathrm{~min}$ (mean $101 \mathrm{~min}, \mathrm{SD} 78 \mathrm{~min}$ ). Only five events of nocturnal hypoglycemia (all with ECG recording) were stated as symptomatic (sweating, trembling).

TAB LE 1 Baseline and laboratory participant characteristics

\begin{tabular}{|lcc|}
\hline & Mean (SD) & Median (range) \\
\hline Age, years & & \\
\hline Male, $n / \%$ & $13.5(2.5)$ & $13.9(8.1-17.6)$ \\
\hline HbA1c, \% & $14 / 56 \%$ & \\
\hline HbA1c, mmol/mmol & $7.8(1.4)$ & $7.5(6.3-12.9)$ \\
\hline Insulin regimen: CSII, $n / \%$ & $62.1(14.8)$ & $58.5(45.4-117.5)$ \\
\hline Insulin, U/kg/d & $14 / 56 \%$ & \\
\hline Pre-study potassium, mmol/I & $0.9(0.2)$ & $0.9(0.5-1.4)$ \\
\hline Pre-study calcium, mmol/I & $2.7(0.5)$ & $4.6(3.8-5.9)$ \\
\hline Pre-study magnesium, mmol/I & $0.8(0.05)$ & $0.8(0.7-0.9)$ \\
\hline
\end{tabular}




\subsection{Arrhythmia}

No cardiac arrhythmias were observed.

\section{5 | Cardiac repolarization/QTc}

\subsection{1 | QTcintervals and nocturnal hypoglycemia}

Mean QTc during nocturnal hypoglycemia (412 $\pm 15 \mathrm{~ms}$ ) was longer compared to euglycemia $(405 \pm 18 \mathrm{~ms}, p=0.005)$, Figure 1 . This

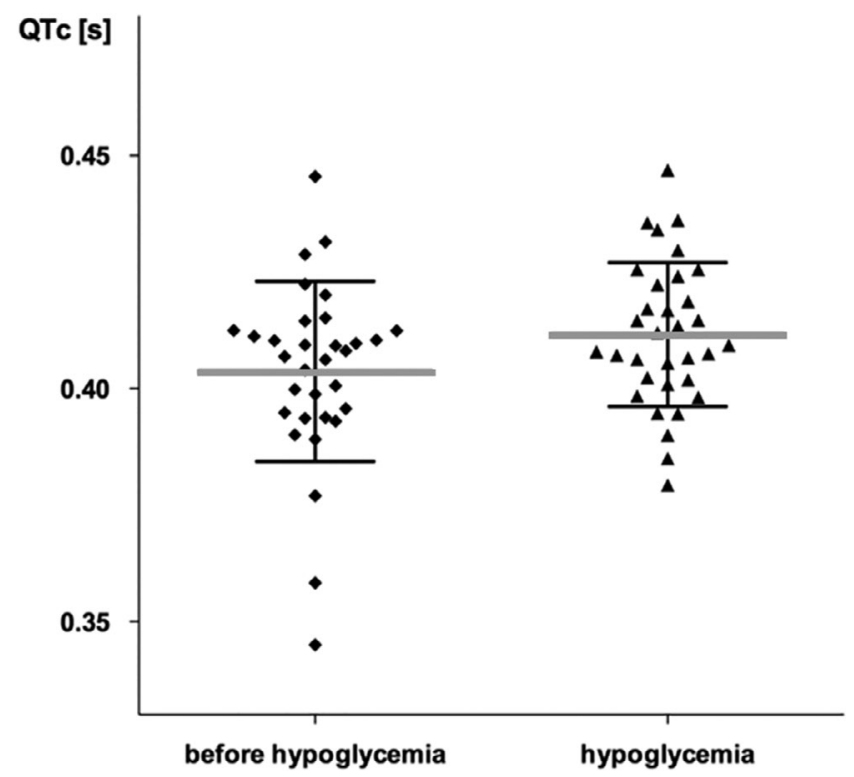

FIGURE 1 QTc(s) during hypoglycemic episodes, compared to preceding intervals of the same duration (thick gray line represents mean, black bars represent \pm 1 SD) change was not dependent on age $(p=0.99)$, sex $(p=0.06)$, HbA1c $(p=0.76)$ or duration of diabetes disease $(p=0.31)$. No significant impact on QTc lengthening was observed for the duration or severity (nadir) of the hypoglycemic episode ( $p=0.90$ and 0.38 , respectively). However, QTc prolongation was more pronounced with lower magnesium levels $(p=0.045)$.

\subsection{2 | Overall correlation of QTc intervals and} glucose levels

QTc duration and interstitial glucose were inversely correlated: QTc intervals were longer with lower glucose levels ( $p$-values between $<0.001$ and 0.02 depending on lag times; Figure 2). This effect was not observed when QTc intervals were correlated to simultaneously recorded glucose values, but only detectable from a time delay of $15 \mathrm{~min}$ on. The strongest effect of glucose level on QTc intervals was seen after 105 min: per every $1 \mathrm{mmol} / \mathrm{L}$ fall in glucose, QTc increased by $0.96 \mathrm{~ms}(p=<0.001$, Figure S1), after which the association faded again.

\section{6 | Heart rate variability}

Changes in heart rate and HRV parameters are displayed in Table 2 and Figure 3.

Heart rate increased from the $15 \mathrm{~min}$ interval before hypoglycemia (mean $69 \pm 9 \mathrm{bpm}$ ) to the 15 min interval after nadir (mean 72 $\pm 12 \mathrm{bpm}, p=0.040$, Figure 3A).

Similarly, several HRV parameters changed during hypoglycemia, that is, from $15 \mathrm{~min}$ before hypoglycemia to the interval after nadir of the hypoglycemic event. RMSSD decreased from $88 \pm 57 \mathrm{~ms}$ to 73 $\pm 43 \mathrm{~ms}$ ( $p=0.039$, Figure $3 \mathrm{~B}$ ), as well as HF power (given in normalized units): from $54 \pm 17$ to $47 \pm 19$ ( $p=0.041$, Figure 3D).

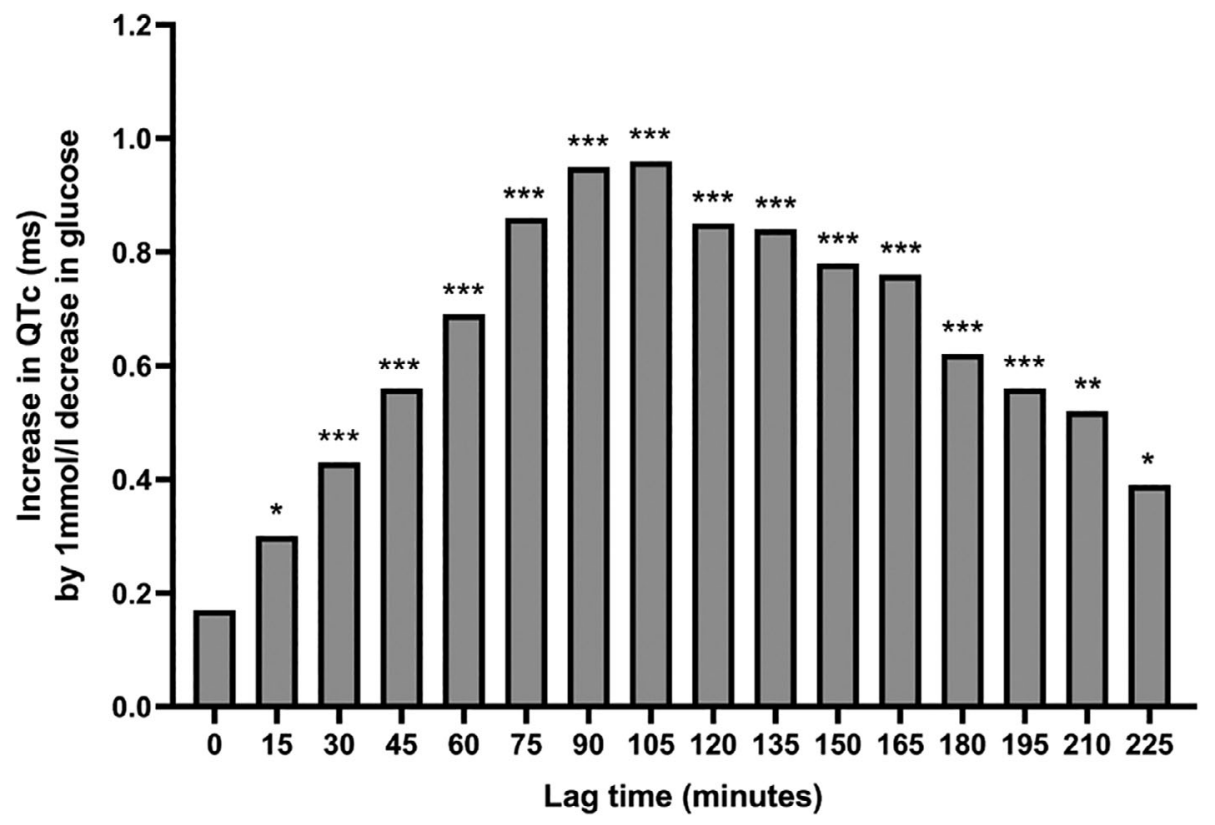

FIGURE 2 Associations of QTc and interstitial glucose for different lag times, from concurrently measured ( $0 \mathrm{~min}$ ) to glucose preceding QTc (up to $225 \mathrm{~min}$ ). Bars represent the increase in QTc (ms) for each $1 \mathrm{mmol} / \mathrm{L}$ decrease in glucose. ${ }^{*} p<0.05,{ }^{* *} p<0.01$, ${ }^{* * *} p<0.001$ 
TAB LE 2 Heart rate and selected HRV parameter changes during hypoglycemia

\begin{tabular}{|c|c|c|c|c|c|c|c|c|}
\hline Variable & $\begin{array}{l}\text { Before } \\
\text { hypoglycemia } \\
\text { mean (SD) }\end{array}$ & $\begin{array}{l}\text { Hypoglycemia } \\
\text { start mean (SD) }\end{array}$ & $\begin{array}{l}\text { Before nadir } \\
\text { mean (SD) }\end{array}$ & $\begin{array}{l}\text { After nadir } \\
\text { mean (SD) }\end{array}$ & $p$-Value ${ }^{a}$ & $\begin{array}{l}\text { Hypoglycemia } \\
\text { end mean (SD) }\end{array}$ & $\begin{array}{l}\text { After } \\
\text { hypoglycemia } \\
\text { mean (SD) }\end{array}$ & $p$-Value ${ }^{b}$ \\
\hline RMSSD, ms & $88(57)$ & $80(45)$ & $79(42)$ & $73(43)$ & 0.040 & $77(47)$ & $85(45)$ & 0.080 \\
\hline SDRR, ms & $97(39)$ & $98(36)$ & $95(32)$ & $99(33)$ & 0.99 & $102(34)$ & $99(39)$ & 0.768 \\
\hline HF, nu & 54 (17) & 50 (17) & $51(17)$ & 47 (19) & 0.041 & 47 (17) & 49 (14) & 0.538 \\
\hline $\mathrm{LF} / \mathrm{HF}$ & $1.0(0.7)$ & $1.2(0.8)$ & $1.2(0.7)$ & $1.6(1.3)$ & 0.024 & $1.5(1.3)$ & $1.2(0.7)$ & 0.116 \\
\hline Total, $\mathrm{ms}^{2}$ & 4150 (3163) & 2930 (1854) & 3359 (2487) & $4572(2765)$ & 0.17 & 4896 (8980) & $3720(2488)$ & 0.182 \\
\hline
\end{tabular}

Note: Parameters calculated for different intervals of $15 \mathrm{~min}$. Values given in mean and SD.

Abbreviations: HF, high frequency power, given in normalized units; HRV, heart rate variability; LF, low frequency power, given in normalized units; pRR50, percentage of subsequent RR intervals that differ more than $50 \mathrm{~ms}$; RMSSD, square root of the mean standard differences of successive RR intervals; SDRR, standard deviation of the RR interval.

${ }^{a}$ Represents linearly tested change from before hypoglycemia to after nadir.

${ }^{\mathrm{b}}$ Represents linearly tested change from after nadir to after hypoglycemia.

FIGURE 3 Changes of heart rate and HRV during hypoglycemia. (A) Heart rate (bpm). (B) RMSSD (ms; square root of the mean standard differences of successive RR Intervals) (C) LF (normalized units, nu), low frequency power. (D) HF (nu) high frequency power. HR and LF increase from before hypoglycemia to after nadir with no further change thereafter. RMSSD and HF decrease in the same time intervals. HF, high frequency power; HRV, heart rate variability; LF, low frequency power
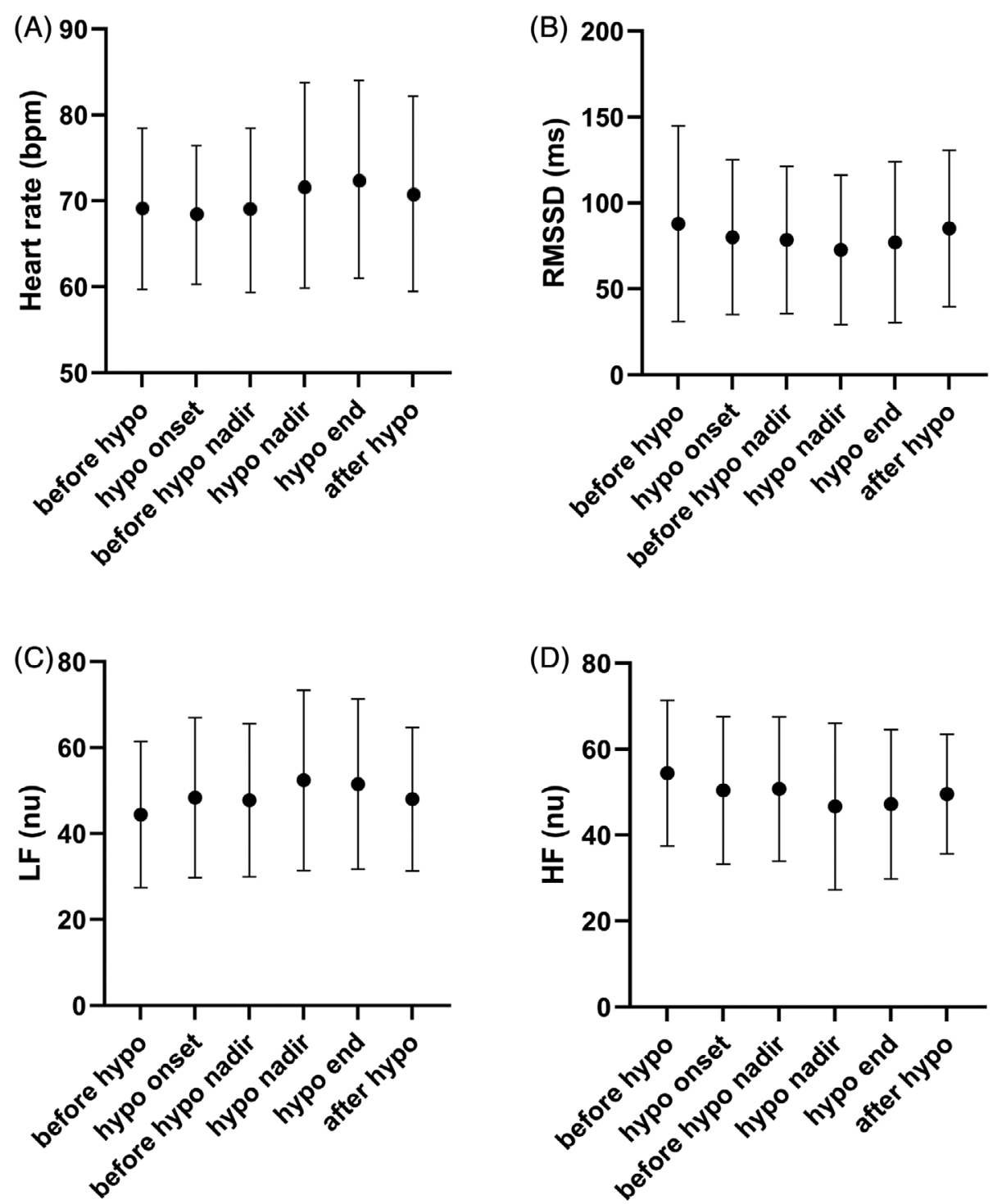
LF power (given in normalized units) increased from $44 \pm 17$ to $52 \pm 21$ ( $p=0.048 \mathrm{~s}$. Figure $3 \mathrm{C}$ ), as did the relation LF/HF (from $1.0 \pm 0.7$ to $1.6 \pm 1.3, p=0.024)$.

These changes in HRV variables were not dependent on age, sex, HbA1c, diabetes duration, the duration or nadir of hypoglycemia.

No significant changes in the intervals after nadir to end of hypoglycemia and after hypoglycemia were observed. Furthermore, no significant changes during hypoglycemia were observed for SDRR, pRR50 or total power.

\section{4 | DISCUSSION}

This study shows that nocturnal hypoglycemia causes mild but significant prolongation of corrected QT intervals in children with T1D, and that QTc intervals correlate directly with glucose levels most pronounced toward the end of the hypoglycemic episode. Furthermore, HRV parameters changed significantly during hypoglycemia reflecting autonomic modulation. To our knowledge, this is the first study that evaluates cardiac repolarization and heart rate variability during episodes of spontaneous nocturnal hypoglycemia in children with T1D in the home setting.

The prolongation of QTc intervals was significant, however, no abnormal QTc values (i.e., longer than $460 \mathrm{~ms}$ ) were seen. The changes in QTc intervals seen in our study were rather small. This could be associated to the fact that ECG was only measured through the night. It is known, that sympatho-adrenergic response is reduced by sleep and horizontal position. ${ }^{19}$

The extent of QTc lengthening in our study is comparable to an earlier study in children with diabetes, ${ }^{20}$ where glucose was measured in serial venous and not CGM overnight samplings.

Several studies in adults reported a lengthening of QTc intervals during spontaneous nocturnal hypoglycemia, however with variable extent and different methods, ${ }^{6,8,9}$ Table S1.

A recent study differentiating between hypoglycemia at daytime and nighttime in 37 adults with diabetes mellitus showed a moderate change in QTc interval during the day and an even smaller change at nighttime. ${ }^{9}$

Gill et al used a setting comparable to our study and found a more distinct prolongation of QTC during hypoglycemia compared to euglycemia in 25 adults with type 1 diabetes. $^{8}$

One single study group documented even shorter QTc intervals during nocturnal hypoglycemia compared to euglycemia. ${ }^{21}$ This study however, included only 10 hypoglycemic episodes in six adult patients, and they chose a very short period of 10 heartbeats around the nadir of hypoglycemia for QTc analysis.

The QTc lengthening in our patients was neither dependent of the duration nor the severity of the hypoglycemic episode. This may be explained by the small number of hypoglycemic episodes that were available with concomitant ECG recording.

Because changes in cardiac repolarization are induced by sympatho-adrenergic stimulation due to hypoglycemia, we could have expected a smaller change in QTc in children with longer diabetes duration or history of severe hypoglycemia. However, this was not observed, which could be attributed to the rather short disease duration in children and the small number of participants that experienced severe hypoglycemia in the past. The only moderating factor that influenced QTc prolongation in the context of hypoglycemia was the serum magnesium level. In participants with lower magnesium levels, the increase in QTc was more pronounced. This is of relevance, as serum magnesium levels are easy to measure and substitution is uncomplicated, not expensive and with few side effects. The effect of magnesium on the hypoglycemia induced QTc lengthening is even more significant given that children with type 1 diabetes were found to have lower magnesium levels than healthy controls. ${ }^{22}$

In addition to a lengthening of QTc intervals during nocturnal hypoglycemia in children, QTc intervals were correlated to glucose levels. The strongest effect could be seen with a delay of $105 \mathrm{~min}$. This temporal association could support the hypothesis that hypoglycemia counter regulation by sympatho-adrenergic activation is responsible for the QTc lengthening. Further inpatient studies with the possibility to measure adrenal hormones and ECG during nocturnal hypoglycemia would be necessary to prove this hypothesis.

Furthermore, the study found significant changes in HRV parameters reflecting autonomic modulation during hypoglycemia.

Of note, a significant change in HRV parameters occurred already $15 \mathrm{~min}$ before low sensor glucose levels were detected. This is in accordance with results of a study in adults with diabetes that combined HRV measures from Holter ECGs with CGM and showed that hypoglycemia could be predicted earlier and with a higher sensitivity than by CGM alone. ${ }^{14,23}$ Two other studies in adults report hypoglycemia induced changes in HRV parameters. ${ }^{9,24}$ In contrast to our results, they reported lower values for LF in hypoglycemia. However, their approach was not comparable to our study: HRV changes were not analyzed before the onset and throughout hypoglycemia, but they compared a 5 min period in hypoglycemia with an euglycemic period in another night.

A recent publication presented data of HRV changes (LF/HF and RMSSD) before the onset of hypoglycemia measured by a HealthPatch in 23 adults. Similar to our study, LF/HF increased significantly, whereas RMSSD decreased. ${ }^{13}$

So far, HRV during nocturnal hypoglycemia has not been studied in children. However, one recent study reported a change in heart rate before nocturnal hypoglycemia compared to euglycemia. ${ }^{25}$

Strengths of this study are the continuous recording of highquality long-term ECG, the gold standard for HRV analysis combined with glucose monitoring for five consecutive nights. A second strength is the real-life setting of the study. Furthermore, young patients from school age were included.

Limitations are the small sample size of 25 participants. However, other studies in this field report data of a similar sample size. As the study was performed in the home setting, and ECG recording was initiated by the participants/parents every night, technical problems occurred in some participants resulting in incomplete recordings. Confirmation of low sensor glucose levels by capillary blood glucose 
measurements would have been desirable. However, we mostly used blinded CGM and only analyzed nighttime glucose profiles, thus hypoglycemia was not noticed by the patients in most cases. As the measurements were not carried out in the hospital, Magnesium levels could not be measured at the moment of hypoglycemia, but at the first day, before the start of the measurement period. Therefore, some variations in Magnesium levels during the study cannot be ruled out. However, a rather low intraindividual variation in serum Magnesium levels has been reported in different studies (1.93 and 3.2\%). ${ }^{26,27}$

In conclusion, this study documents that nocturnal hypoglycemia has a QTc prolonging effect in children with diabetes. This QTc alteration was not clinically relevant in our patient group. However, one can assume that in predisposed subjects (i.e., intrinsic QT prolongation due to genetic polymorphisms in ion channel genes, electrolyte imbalances and/or co-medications which prolong QT intervals), QTc prolongation aggravated due to hypoglycemia might become clinically significant. Such patients should be identified and risk constellations avoided.

This is particularly important, as there is evidence, that children with T1D have longer QTc intervals than healthy controls. ${ }^{28-30} \mathrm{~A}$ recent study even found prolonged QTc intervals (>450 ms) in onethird of children with T1D. ${ }^{25}$ A superimposed additional QTC prolonging effect of hypoglycemia could be critical in those children. Remarkably, not only hypoglycemia but also diabetic ketoacidosis (DKA) seems to have an impact on QTc intervals: several studies report mean QTc intervals during DKA between 447 and $458 \mathrm{~ms}$ with prolonged QTc in 31 up to $53 \%$ of patients. ${ }^{31-34}$

It may therefore be advisable to perform occasional ECG recordings in children with diabetes, as treatment with beta-blockers could reduce the risk of cardiac arrhythmia. ${ }^{35}$ In these patients at risk, hypoglycemia should be avoided even more aggressively. Avoidance of hypoglycemia is however more difficult in children, because they may not be able to recognize or communicate symptoms of hypoglycemia due to their age. $^{36}$ In addition, eating behavior and physical activity are mostly unforeseeable. Children with T1D would therefore particularly benefit from the possibility to earlier detect hypoglycemic events.

The findings of this study suggest that HRV measurements could help to detect hypoglycemia in advance. We could show for the first time in children that HRV parameters change during hypoglycemia and even before hypoglycemia was detected by CGM. Larger studies would be needed to further evaluate the association of HRV and glucose and to confirm these findings.

The recording by Holter ECG used in this study would not be suitable for everyday use and was only performed at nighttime. Comfortable wearable devices measuring heart rate variability are however available, and it may thereby be promising to include measurements of HRV into treatment algorithms for children with diabetes.

\section{ACKNOWLEDGMENTS}

The authors thank all the participants and their families for their interest in the study and their collaboration and Corinne Gantenbein, cardiologic nurse, for the preparation of Holter ECG equipment and the kind instruction of participants. The authors further thank Medtronic for providing two iPro and Enlite sensors.

\section{AUTHOR CONTRIBUTIONS}

Sara Bachmann and Birgit Donner designed and performed the study. Gabor Szinnai, Melanie Hess and Urs Zumsteg recruited patients and reviewed the manuscript. Marie-Anne Burckhardt reviewed and edited the manuscript. Anne Auderset contributed to the analysis as part of her medical master thesis. Kris Denhaerynck analyzed the data and reviewed the manuscript. Sara Bachmann and Birgit Donner wrote the paper.

\section{PEER REVIEW}

The peer review history for this article is available at https://publons. com/publon/10.1111/pedi.13262.

\section{DATA AVAILABILITY STATEMENT}

Research data are not shared.

\section{ORCID}

Sara Bachmann (D) https://orcid.org/0000-0001-7354-0858

Marie-Anne Burckhardt (D) https://orcid.org/0000-0002-6274-5900

\section{REFERENCES}

1. Tu E, Twigg SM, Duflou J, Semsarian C. Causes of death in young Australians with type 1 diabetes: a review of coronial postmortem examinations. Med J Aust. 2008;188:699-702.

2. Secrest AM, Becker DJ, Kelsey SF, Laporte RE, Orchard TJ. Characterizing sudden death and dead-in-bed syndrome in type 1 diabetes: analysis from two childhood-onset type 1 diabetes registries. Diabet Med. 2011;28:293-300.

3. Patel F. Diabetic death in bed: post-mortem determination of hypoglycemia. Med Sci Law. 1994;34:84-87.

4. Tannenberg RJ, Newton CA, Drake AJ. Confirmation of hypoglycemia in the "ded in bed" syndrome, as captured by retrospective continuous glucose monitoring system. Endocr Pract. 2010;167:244-248.

5. Robinson RTCE, Harris ND, Ireland RH, Lee S, Newman C, Heller SR. Mechanisms of abnormal cardiac repolarization during insulin induced hypoglycemia. Diabetes. 2003;52:1469-1474.

6. Robinson RTCE, Harris ND, Ireland RH, Macdonald IA, Heller SR. Changes in cardiac repolarization during clinical episodes of nocturnal hypoglycemia in adults with type 1 diabetes. Diabetologia. 2004;47:312-315.

7. Koivikko ML, Karsikas M, Salmela PI, et al. Effects of controlled hypoglycemia on cardiac repolarisation in patients with type 1 diabetes. Diabetologie. 2008;51:426-435.

8. Gill GV, Woodward A, Casson IF, Weston PJ. Cardiac arrhythmia and nocturnal hypoglycemia in type 1 diabetes-the "dead in bed" syndrome revisited. Diabetologia. 2009;52:42-45.

9. Novodvorsky P, Bernjak A, Chow E, et al. Diurnal differences in risk of cardiac arrhythmias during spontaneous hypoglycemia in young people with type 1 diabetes. Diabetes Care. 2017;40:655-662.

10. Kacheva S, Karges B, Göller K, Marx N, Mischke K, Karges W. QT prolongation caused by insulin-induced hypoglycemia-an interventional study in 119 individuals. Diabetes Res Clin Pract. 2017;123:165-172.

11. Bachmann S, Hess M, Martin-Diener E, Denhaerynck K, Zumsteg U. Nocturnal hypoglycemia and physical activity in diabetic children: new insights by continuous glucose monitoring. Diabetes Care. 2016; 39(7):e95-e96.

12. Bilchick KC, Berger RD. Heart rate variability. J Cardiovasc Electrophysiol. 2008;17(6):691-694.

13. Bekkink MO, Koeneman M, De Galan BE, Bredie SJ. Early detection of hypoglycemia in type 1 diabetes using heart rate variability measured by a wearable device. Diabetes Care. 2019;42(4):689-692. 
14. Cichosz SL, Frystyk J, Tarnow L, Fleischer J. Combining information of autonomic modulation and CGM measurements enables prediction and improves detection of spontaneous hypoglycemic events. $J$ of Diabetes Sci Technol. 2015;9:132-137.

15. Welsh JB, Kaufman FR, Lee SW. Accuracy of the Sof-sensor glucose sensor with the iPro calibration algorithm. J Diabetes Sci Technol. 2012;6(2):475-476.

16. Nakamura K, Balo A. The accuracy and efficacy of the Dexcom G4 platinum continuous glucose monitoring system. J Diabetes Sci Technol. 2015;9(5):1021-1026.

17. Keenan DB, Mastrototaro JJ, Zisser H, et al. Accuracy of the Enlite 6-day glucose sensor with guardian and Veo calibration algorithms. Diabetes Technol Ther. 2012 Mar;14(3):225-231.

18. Task force of the European Society of Cardiology, The North American Society of Pacing Electrophysiology. Heart rate variability. Standards of measurement, physiolocigal interpretation and clinical use. Circulation. 1996;93:1043-1065.

19. Jones TW, Porter P, Sherwin RS, et al. Decreased epinephrine response to hypoglycemia during sleep. N Engl J Med. 1998;338:1656-1662.

20. Murphy NP, Ford-Adams ME, Ong KK, Harris ND, Keane SM, Davies SM. Prolonged cardiac repolarization during spontaneous nocturnal hypoglycemia in children and adolescents with type 1 diabetes. Diabetologia. 2004;47:1940-1947.

21. Koivikko ML, Kenttä T, Salmela PI, Huikuri HV, Perkiömäki JS. Changes in cardiac repolarisation during spontaneous nocturnal hypoglycemia in subjects with type 1 diabetes: a preliminary report. Acta Diabetol. 2017;54(3):251-256.

22. Lin CC, Tsweng GJ, Lee CF, et al. Magnesium, zinc, and chromium levels in children, adolescents and young adults with type 1 diabetes. Clin Nutr. 2016;35(4):880-884.

23. Cichosz SL, Frystyk J, Hejlesen OK, Tarnow L, Fleischer J. A novel algorithm for prediction and detection of hypoglycemia based on continuous glucose monitoring and heart rate variability in patients with type 1 diabetes. J Diabetes Sci Technol. 2014;8(4):731-737.

24. Koivikko ML, Tulppo MP, Kiviniemi AM, et al. Autonomic cardiac regulation during spontaneous nocturnal hypoglycemia in patients with type 1 diabetes. Diabetes Care. 2012;35(7):1585-1590.

25. Calcaterra V, Bosoni P, Sacchi L, et al. Continuous glucose and heart rate monitoring in young people with type 1 diabetes: an exploratory study about perspectives in nocturnal hypoglycemia detection. Metabolites. 2020;11(1):5.

26. Djurhuus MS, Gram J, Petersen PH, Klitgaard NA, Bollerslev J, BeckNielsen $\mathrm{H}$. Biological variation of serum and urinary magnesium in apparently healthy males. Scand J Clin Lab Invest. 1995;55(6):549-558.
27. Valero-Politi J, Ginard-Salvá M, González-Alba JM. Annual rhythmic and non-rhythmic biological variation of magnesium and ionized calcium concentrations. Clin Chem Lab Med. 2001;39(1):45-49.

28. Galli-Tsinopoulou A, Chatzidimitriou A, Kyrgios I, Rousso I, Varlamis G, Karavanaki K. Children and adolescents with type 1 diabetes mellitus have a sixfold greater risk for prolonged QTc interval. J Pediatr Endocrinol Metab. 2014;27(3-4):237-243.

29. El-Walili T, Adel HM, Abdel Meguid SE, Elsayed AIK. Assessment of corrected QT interval in children with type 1 diabetes mellitus. Alexandria J Pediatr. 2019;32:51-54.

30. Suys BE, Huybrechts SJ, De Wolf D, et al. QTc interval prolongation and QTc dispersion in children and adolescents with type 1 diabetes. J Pediatr. 2002;141(1):59-63.

31. Perez MM, Medar S, Quigley L, Clark BC. QTc prolongation in pediatric patients with diabetic ketoacidosis. J Pediatr. 2021;228: 235-239.

32. Aygün D, Aygün F, Nişli K, Baş F, Çıtak A. Electrocardiographic changes in children with diabetic ketoacidosis and ketosis. Turk Pediatri Ars. 2017;52(4):194-201.

33. Youssef OI, Farid SM. QTc and QTd in children with type 1 diabetes mellitus during diabetic ketoacidosis. ISRN Pediatr. 2012;4:619107.

34. Kuppermann N, Park J, Glatter K, Marcin JP, Glaser NS. Prolonged QT interval corrected for heart rate during diabetic ketoacidosis in children. Arch Pediatr Adolesc Med. 2008;162(6):544-549.

35. Abu-Zeitone A, Peterson DR, Polonsky B, McNitt S, Moss AJ. Efficacy of different beta-blockers in the treatment of long QT syndrome. J Am Coll Cardiol. 2014;64(13):1352-1358.

36. Gonder-Frederick L, Zrebiec J, Bauchowitz A, et al. Detection of hypoglycemia by children with type 1 diabetes 6 to 11 years of age and their parents: a field study. Pediatrics. 2008;121(3):e489-e495.

\section{SUPPORTING INFORMATION}

Additional supporting information may be found in the online version of the article at the publisher's website.

How to cite this article: Bachmann S, Auderset A,

Burckhardt $\mathrm{M}-\mathrm{A}$, et al. Autonomic cardiac regulation during spontaneous nocturnal hypoglycemia in children with type 1 diabetes. Pediatr Diabetes. 2021;22(7):1023-1030. doi: 10.1111/pedi.13262 